KLEINE TEXTE FUR THEOLOGISCHE UND PHILOLOGISCHE VORLESUNGEN UND UBUNGEN

HERAUSGEGEBEN VON HANS LIETZMANN

\title{
SUPPLEMENTUM LYRICUM
}

\section{NEUE BRUCHSTÜCKE}

VON

ARCHILOCHUS ALCAEUS SAPPHO CORINNA PINDAR

AUSGEWÄHLT UND ERKLÄRT

voN

DR. ERNST DIEHL

A. O. PROFESSOR IN JENA

ZWEITE AUFLAGE

BONN

A. MARCUS UND E. WEBER'S VERLAG

1910 